\title{
A Rare Case of Multicentric Liposarcoma and Associated Reproductive Aberrations in a Nonpregnant Holstein-Friesian Crossbred Cow
}

\author{
Pankaj Sood, ${ }^{1}$ Parvesh Kumar, ${ }^{1}$ Sarvesh Kumar, ${ }^{2}$ Adarsh Kumar, ${ }^{3}$ \\ Rajesh Kumar Asrani, ${ }^{2}$ and Madhumeet Singh ${ }^{1}$ \\ ${ }^{1}$ Department of Veterinary Gynaecology and Obstetrics, DGCN College of Veterinary and Animal Sciences, CSK HPKV, \\ Palampur 176 062, India \\ ${ }^{2}$ Department of Veterinary Pathology, DGCN College of Veterinary and Animal Sciences, CSK HPKV, Palampur 176 062, India \\ ${ }^{3}$ Department of Veterinary Surgery and Radiology, DGCN College of Veterinary and Animal Sciences, CSK HPKV, \\ Palampur 176 062, India
}

Correspondence should be addressed to Pankaj Sood, psoodhpkv@yahoo.com

Received 27 July 2011; Accepted 21 August 2011

Academic Editors: C. Gutierrez, F. Mutinelli, and H.-M. Park

Copyright () 2011 Pankaj Sood et al. This is an open access article distributed under the Creative Commons Attribution License, which permits unrestricted use, distribution, and reproduction in any medium, provided the original work is properly cited.

A five-and-a-half-year-old Holstein-Friesian crossbred cow was repeatedly treated for termination of overgestation, but without any success. On laparotomy, the site indicating fetal-like ballottement on transrectal palpation was occupied by three to four separate caseous and lobulated masses with adhered uterine horns in the omentum. Separation of the utero-omental adhesions retrieved normal-sized uterine horns into pelvic cavity. The right ovary was cystic. Ultrasonography detected another growth in the ventral abdominal musculature. Histopathologically, the omental growths suggested a pleomorphic type of liposarcoma. Presence of omental and muscular liposarcoma along with deep uterine-omental adhesions and a cystic ovary makes this paper a unique addition to the literature.

Benign lipomas of adipose tissue in cattle are not uncommon. They are usually found single and may be present at a variety of sites $[1,2]$. In contrast, malignant and metastatic liposarcomas are a rare occurrence in animals [3] and have been documented in two separate reports in cattle $[4,5]$. The latter has also been reported in the companion $[6,7]$ and wild [8] animals including reptiles [9], rat [10], and chameleon [11]. The present paper adds to the literature the presence of a multicentric and metastatic liposarcoma in omentum and ventral abdominal musculature along with an associated uterine-mesenteric adhesions and cystic ovary in a cow.

A five-and-a-half-year-old Holstein-Friesian crossbred cow was referred to the State Clinical and Referral Hospital of Veterinary College, Palampur, India, with a history of failure of termination of overgestation (9.75 months) using hormones. The induction protocol comprised of a combination of $500 \mathrm{mcg}$ cloprostenol (Clostenol; Sarabhai
Zydus, India) intramuscularly and $40 \mathrm{mg}$ dexamethasone sodium phosphate (Decamycin; Ranbaxy Laboratories Limited, India) intravenously, repeated once at an interval of five days. The cow was presented in the Clinics one week after the second hormonal treatment.

On general examination, the cow was alert, active and normal in feeding, defecation, and urination. There were no external indications of impending parturition. On transrectal palpation, the external os was palpable deep in the abdomen, whereas the uterine horns could not be appreciated. A distinct fetal bump kind of ballottement was felt deep ahead of the cervix in the abdomen.

Speculating a therapeutic failure to induce parturition and relying on the clinical observations, a hormonal treatment comprising of $25 \mathrm{mg}$ dinoprost tromethamine (Lutalyse; Pfizer Animal Health, India) and $40 \mathrm{mg}$ dexamethasone sodium phosphate (Curadex; Concept Pharma, India) was 
given to the cow at the College Clinic. Thereafter, the cow was repeatedly examined every $12 \mathrm{~h}$, but without any change in the genital status. The cow was discharged from the clinics with a caveat of reexamination one month later. Meanwhile, two blood samples were collected at fortnight interval through jugular venipuncture for progesterone estimation using radioimmunoassay.

Reexamination of the cow 30 days later did not reveal any change, and the cow was subjected to laparotomy in lateral recumbency. The incision line was parallel to the milk vein. Exploration of abdomen did not reveal any fetal mass/contents. Instead, the site, indicating fetal-like sensation on transrectal palpation, was occupied by three to four separately localized caseous and lobulated masses of 10$13 \mathrm{~cm}$ diameter in the mesentery (Figure 1). A similar kind of tissue mass was also found in and adjacent to the omentum. Furthermore, during incision, mild adhesions were detected in the musculature adjacent to as well as in peritoneum and omentum. On tracking the cervix, both the uterine horns were found to be adhered to the mesenteric growths in the abdomen. The adhered uterine horns were easily separated manually, following which the entire genitalia retrieved back into pelvic cavity. The two uterine horns were nongravid and of similar size, but the right ovary was three to four times larger than the contra lateral ovary and had a large fluid-filled structure on it. On corroborating the ovarian structure to the plasma progesterone concentrations of 2.4 and $1.5 \mathrm{ng} / \mathrm{mL}$, it was diagnosed to be a luteal cyst.

Incisional biopsies were collected from the multiple sites of the different tissue growths in 10\% buffered formal saline for histopathological investigation [12]. The operative site was sutured in a routine manner and the postsurgical treatment comprised of administration of $3000 \mathrm{mg}$ ceftriaxone and 375 tazobactam (Intacef Tazo; Intas Pharmaceuticals Limited, India) for 5 days and $150 \mathrm{mg}$ meloxicam, $4500 \mathrm{mg}$ paracetamol, and $333 \mathrm{~mL}$ lignocaine (Melonex Plus; Intas Pharmaceuticals Limited, India) for 3 days, by intramuscular route.

Transabdominal ultrasonography $(3.5 \mathrm{MHz})$ of ventral abdomen anterior to navicular region revealed a well-defined hyperechoic, hyperattenuating, and relatively uniform mass (Figure 2). Infiltrating echogenicity and shadowing were the prominent features of the mass. The hyperechoic and hypoechoic foci within the mass represented areas of haemorrhage and necrosis, respectively. The absence of fine echogenic lines with in the mass indicates that it was not a well-differentiated structure.

The cow exhibited uneventful recovery and was discharged after three days.

Histologically, the mass was composed of neoplastic lipocytes containing intracytoplasmic lipid vacuoles of variable sizes, interstitial stroma, and encompassing remnants of mesothelial lining. Cells exhibited large, round to oval nuclei with coarsely clumped chromatin pattern and anisokaryosis. Steatonecrosis was also present (Figure 3). Based on the histological pattern and cell morphology, the findings in the present case were typical for a liposarcoma.

Estrus was observed 35 days after surgery; the cow was diagnosed and treated for endometritis using antibiotics at

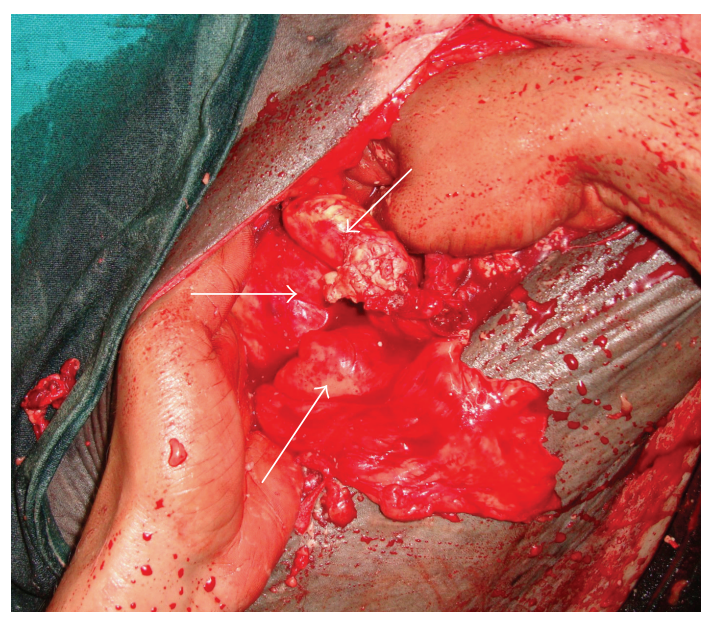

FIGURE 1: Liposarcoma growths (arrows) in omentum detected after laparotomy in a Holstein-Friesian crossbred cow.

farmer's location. Subsequently, the cow was inseminated on two occasions and as per the latest information the cow was nonpregnant four months after the last insemination.

The histopathological features of the tumorous tissue in the present case suggested it to be a pleomorphic type of liposarcoma; the other types reported include well-differentiated and myxoid type of liposarcomas [13]. Presence of lipoblast-like cells in the tumour mass is the most definitive for diagnosis of liposarcoma [14] and helps to differentiate it from almost similar malignant fibrous histocytoma [15].

Liposarcoma is one of the most common malignant mesenchymal tumour and accounts for 20 percent of all sarcomas in humans [16]. Unlike humans, liposarcomas are rare neoplasms in domestic animals [5]. Location of liposarcomas in the mesentery and ventral abdominal muscles in the present case is different from previous reports indicating liposarcomas mainly in the pelvic region, with some growths in the abdominal parietal surfaces, the pleura, the pluck, and the intercostal muscles and throughout the lymphatics of head and neck of a cow [4]. In another cow, liposarcomas were reported in the nasal cavity with local invasions into the oral cavity [5].

Transabdominal sonography confirmed a uniform mass with foci of varying echogenicity. These foci can be areas of haemorrhages and necrosis [17]. The presence of fine echogenic lines within the tumour is a useful sign for diagnosing a well-differentiated liposarcoma. Presence of numerous fine fibrous septa in the lipomatous tumour tissue is thought to be responsible for this interesting phenomenon [18]. In contrast, pleomorphic liposarcoma does not show this type of sign as evident in the present sonogram, which also corroborates to the histopathological findings.

Functional irritation of liposarcomas with the adjacent organs is the cause of adhesion [19] which justifies the uterine-mesenteric adhesions in the present case. However, the descend of the uterus in the abdominal cavity to an extent that it was undetectable by repeated transrectal palpation is inexplicable. On freeing of uterus from adhesions, no external/internal tumorous growths or any other contents 


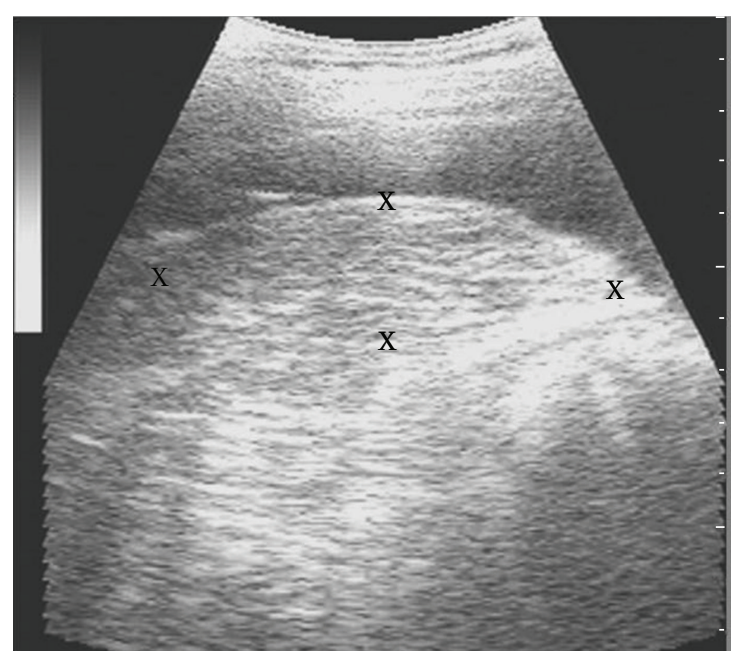

(a)

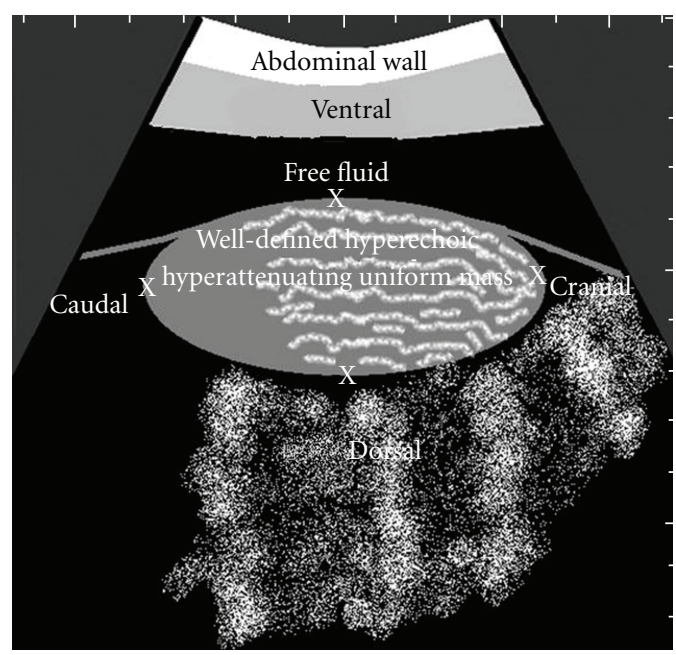

(b)

FIGURE 2: Ventral abdominal sonogram (cranial to navicular region) and its schematic depiction of a well-differentiated hyperechoic hyperattenuating uniform mass with infiltrating echogenicity and hypoechogenic fluid surrounding it.

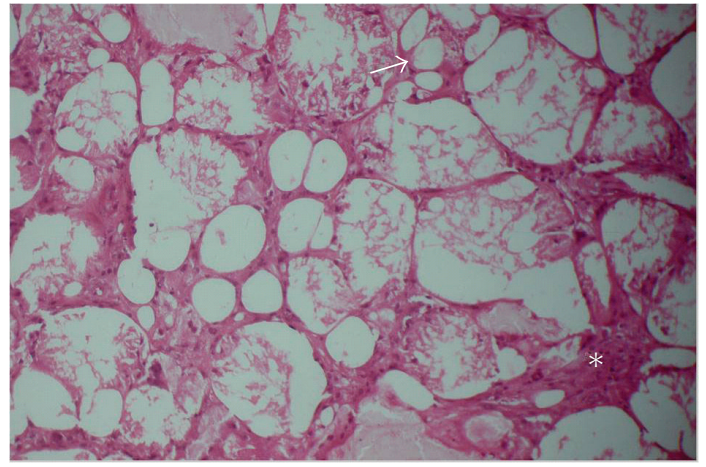

Figure 3: Pleomorphic lipocytes (arrows) encompassed in interstitial stroma and serosal lining (asterix) along with some steatonecrosis $(\mathrm{H} \& \mathrm{E} 132 \mathrm{X})$.

in uterine lumen were detectable, which otherwise could have been the potential reason of uterine descent in the abdomen. Failure to palpate uterine horns and the presence of tumorous mass in mesentery, whose size remained similar on repeated transrectal palpations, resulted in an erroneous diagnosis of presence of fetal mass. Failure of the transrectal ultrasonic probe to reach the tumorous site in abdomen and large size of the cow eluded a conclusive diagnosis of fetal presence. Liposarcomas in the abdominal cavity may be large enough to create intestinal obstruction [19] which, however, was not recorded in the present case due to smaller size of the tumors.

Presence of cystic ovary in the cow could be attributed to endometritis which perturbs the hypothalamo-pituitary axis [20]. The progesterone concentration of $>1 \mathrm{ng} / \mathrm{mL}$ in the two blood samples was suggestive of a luteal cyst, which resolved after endometritis treatment during the post surgery estrous period, and the cow exhibited recurrent estrous cycles.
Most described cases of liposarcomas in animals are considered spontaneous, but some reports have suggested possible inciting agents. Benign liposarcomas surrounding foreign bodies, such as liposarcoma around 1.5-year-old implanted microchip in a dog [21] and liposarcoma around 10 -years-old glass fragment in another dog [22], are on record. However, virus-associated liposarcomas of benign type in a hamster [23] and malignant type in a kitten [24] have also been reported. No attempts to isolate a virus or foreign body in the present paper obscure the probable etiology.

In conclusion, the gross, histopathological, and ultrasonic findings in this case are consistent with a malignant liposarcoma, which misled into the presence of fetus-like mass in deep abdomen; failure to trace the uterine horns due to their adhesions with omentum and presence of cystic ovary were the other features of the affected cow.

\section{References}

[1] T. Gopal and H. W. Leipold, "Lipomeningocele in a calf," Veterinary Pathology, vol. 16, no. 5, pp. 610-612, 1979.

[2] B. O. Ikede, "Bilateral retroperitoneal lipomata in a neonatal calf," Veterinary Record, vol. 98, no. 14, p. 280, 1976.

[3] L. T. Pulley and A. A. Stannard, Tumors in Domestic Animals, University of California Press, Berkeley, Calif, USA, 3rd edition, 1990.

[4] D. W. Piercy, S. J. Furlong, and N. Rudram, "Multicentric liposarcoma in a cow," Veterinary Record, vol. 134, no. 12, pp. 310-311, 1994.

[5] H. Shive, F. Mohammed, J. Osterstock, B. Porter, and J. Mansell, "Liposarcoma in the nasal cavity of a cow," Veterinary Pathology, vol. 43, no. 5, pp. 793-797, 2006.

[6] H. S. Cho, O. J. Kim, and N. Y. Park, "Liposarcoma in the lung of a poodle dog," Korean Journal of Veterinary Research, vol. 3, no. 46 , pp. 263-265, 2006. 
[7] F. I. Wang, S. L. Liang, H. L. Eng, C. R. Jeng, and V. F. Pang, "Disseminated liposarcoma in a dog," Journal of Veterinary Diagnostic Investigation, vol. 17, no. 3, pp. 291-294, 2005.

[8] N. Aihara and Y. Une, "Pleomorphic liposarcoma of the intrathoracic cavity in a meerkat, Suricata suricatta," Journal of Veterinary Medical Science, vol. 71, no. 5, pp. 685-688, 2009.

[9] N. Schmid-Brunclik, S. Simova-Curd, M. Kaufmann-Bart, M. Gassmann, and J. M. Hatt, "Liposarcoma in a veiled chameleon (Chamaeleo calyptratus)," Journal of Herpetological Medicine and Surgery, vol. 4, no. 17, pp. 132-135, 2008.

[10] Y. Minato, H. Takada, H. Yamanaka et al., "Pleomorphic liposarcoma in an aged rat," The Japanese Journal of Veterinary Science, vol. 48, no. 2, pp. 429-432, 1986.

[11] N. S. Brunclik, S. C. Stefka, M. Kaufmann-Bart, G. Max, and H. J. Michel, "Liposarcoma in a veiled chameleon, Chamaeleo calyptratus," Journal of Herpetological Medicine and Surgery, vol. 4, no. 17, pp. 132-135, 2008.

[12] L. G. Luna, Manual of Histologic Staining Methods of the Armed Forces Institute of Pathology, WB Saunders, Philadelphia, Pa, USA, 3rd edition, 1972.

[13] M. J. Hendrick, E. A. Mahaffey, and F. M. Moore, "Histological classification of mesenchymal tumors of skin and soft tissues of domestic animals," in World Health Organization International Histological Classification of Tumors of Domestic Animals, p. 19, Armed Forces Institute of Pathology, Washington, DC, USA, 2nd edition, 1998.

[14] T. Minase, "Tumors of connective tissue," in Electron Microscopy of Tumors, T. Onoe, Ed., pp. 61-68, Nanzando, Tokyo, Japan, 1982.

[15] T. Shimoda, H. Yamashita, M. Furusato et al., "A light and electron microscopic study with comments on their relation to malignant fibrous histiocytoma and angiosarcoma," Acta Pathology Japan, vol. 5, no. 30, pp. 779-797, 1980.

[16] A. P. Dei Tos, "Liposarcoma: new entities and evolving concepts," Annals of Diagnostic Pathology, vol. 4, no. 4, pp. 252-266, 2000.

[17] B. Khan, D. J. Sherlock, G. Wilson, and D. Butterworth, "Sonographic appearance of primary liver liposarcoma," Journal of Clinical Ultrasound, vol. 1, no. 29, pp. 44-47, 2001.

[18] H. Ishida, H. Naganuma, K. Konno et al., "Retroperitoneal liposarcoma sonographic findings," Abdominal Imaging, vol. 5, no. 25, pp. 554-558, 2000.

[19] K. V. F. Jubb, P. C. Kennedy, and N. Palmer, Pathology of Domestic Animals, vol. 2, Academic Press Incorporation, New York, NY, USA, 5th edition, 2007.

[20] S. J. Roberts, "Infertility in the cow," in Veterinary Obstetrics and Genital Diseases (Theriogenology), pp. 481-483, David \& Charles, Devon, UK, 3rd edition, 1986.

[21] M. Vascellari, F. Mutinelli, R. Cossettini, and E. Altinier, "Liposarcoma at the site of an implanted microchip in a dog," Veterinary Journal, vol. 168, no. 2, pp. 188-190, 2004.

[22] P. E. McCarthy, C. S. Hedlund, R. S. Veazy, J. PrescottMathews, and D. Y. Cho, "Liposarcoma associated with a glass foreign body in a dog," Journal of the American Veterinary Medical Association, vol. 209, no. 3, pp. 612-614, 1996.

[23] M. H. Moar, W. F. H. Jarrett, and B. W. O’Neill, "Viral DNA sequences detected in a hamster liposarcoma induced by bovine papillomavirus type 4," Journal of General Virology, vol. 67, no. 1, pp. 187-190, 1986.

[24] L. C. Stephens, C. C. Tsai, G. L. Raulston, J. H. Jardine, and W. F. MacKenzie, "Virus-associated liposarcoma and malignant lymphoma in a kitten," Journal of the American Veterinary Medical Association, vol. 183, no. 1, pp. 123-125, 1983. 

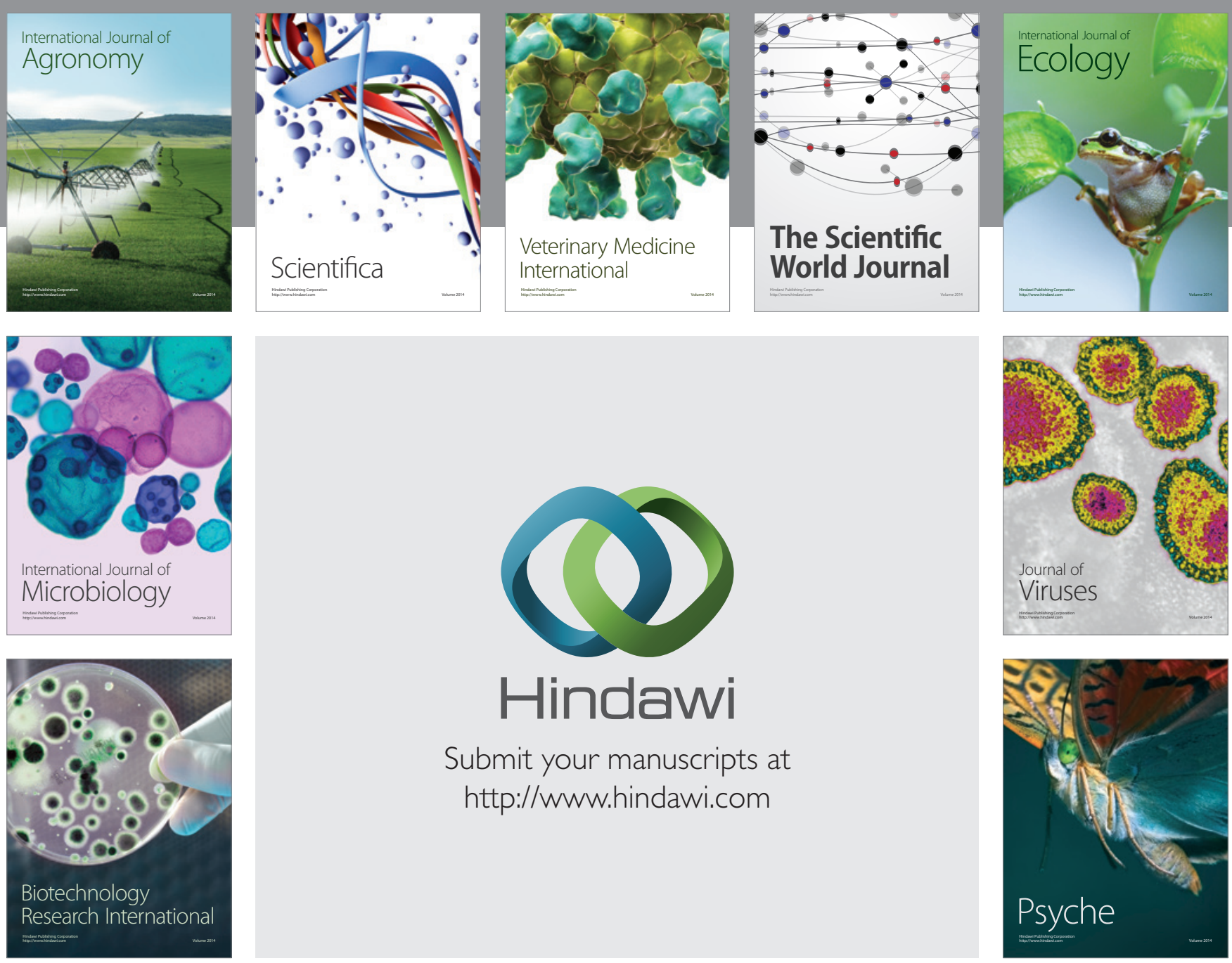

Submit your manuscripts at

http://www.hindawi.com
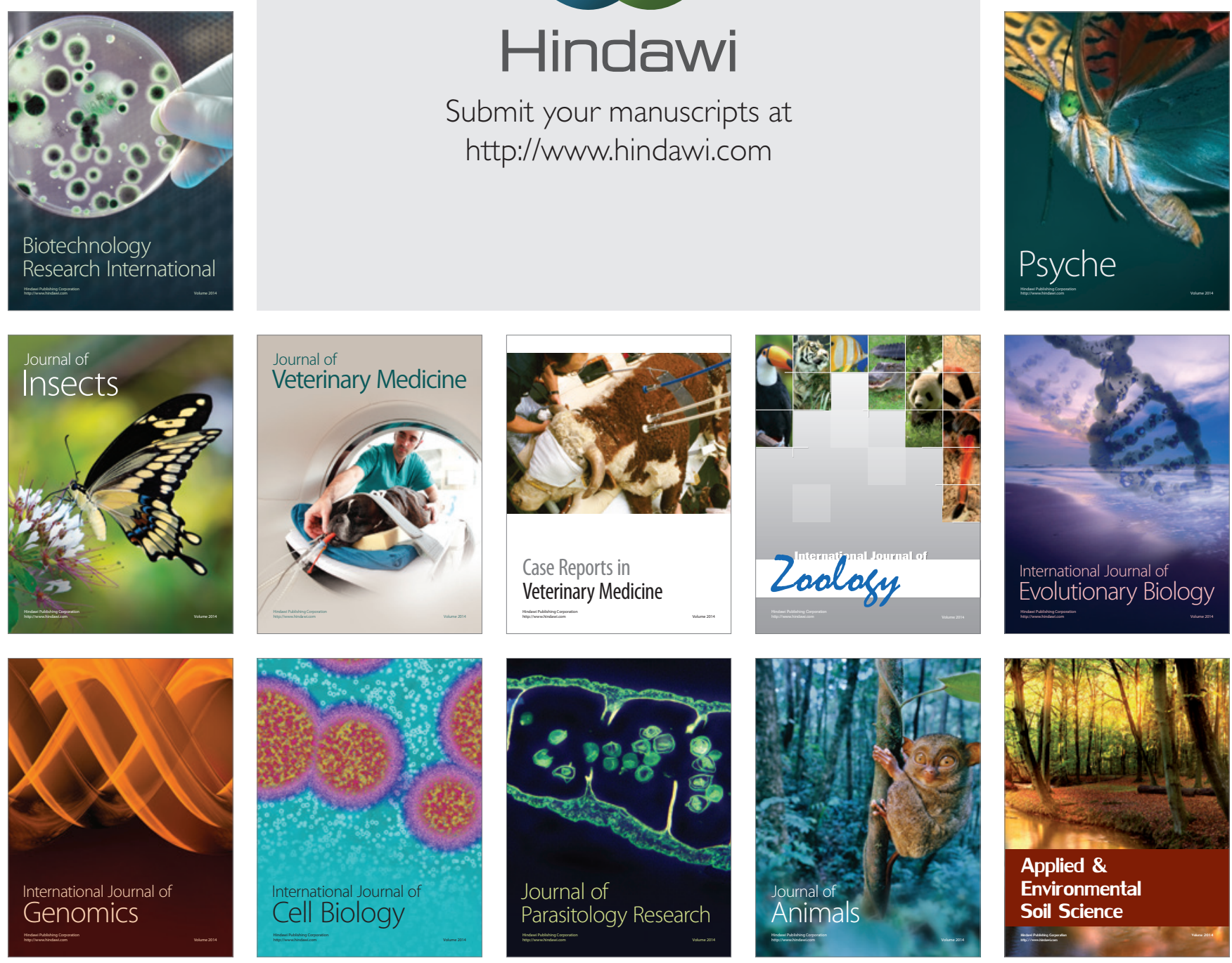